\title{
Study of the Dielectric Behavior as a Function of the Frequency at Different Temperatures on the Sample $\mathrm{Pr}_{0.525} \mathrm{Y}_{0.075} \mathrm{Ca}_{0.1} \mathrm{Sr}_{0.3} \mathrm{Mn}_{0.975} \mathrm{Fe}_{0.025} \mathrm{O}_{3}$
}

\section{Baazaoui ${ }^{1 *}$, A Moulahi' ${ }^{2}$ N Hamdaoui ${ }^{3}$ and M Oumezzine ${ }^{1}$}

${ }^{1}$ Laboratory of Physical Chemistry of Materials, Department of Physics, Faculty of Sciences of Monastir, University of Monastir, Tunisia

${ }^{2}$ Department of Chemistry, Al-Wajha University College, Saudi Arabia

${ }^{3}$ University of Sousse, ISITCom, Tunisia

\begin{abstract}
Structural and dielectric properties of $\operatorname{Pr}_{0.525} \mathrm{Y}_{0.075} \mathrm{Ca}_{0.1} \mathrm{Sr}_{0.3} \mathrm{Mn}_{0.975} \mathrm{Fe}_{0.025} \mathrm{O}_{3}$ manganite system are investigated. The XRD analysis confirms that all compounds crystallized in a rhombohedral structure ( $R \overline{3} c$ space group). Using complex impedance spectroscopy, we confirm that our sample proves a semiconductor behavior which is governed by the increase of the conductance as function of temperature. From the complex impedance spectra, we conclude that the electrical mechanism for each temperature indicate a non-Debye type relaxation behavior. The achieved activation energy is found to be $E_{a}=0.117 \mathrm{ev}$. Indeed, we proved that the abrupt decrease of dielectric lost a $\delta$ with frequency is related to the decrease of the grain resistance at high frequencies.
\end{abstract}

\section{Keywords}

Impedance spectroscopy, Electrical conductivity, Complex impedance spectra, Dielectric properties

\section{Introduction}

Manganite perovskite have an important impact on technological advances due to their wide range of magnetic and transport properties [1]. Based on the comprehension of their fundamental physical properties, such as electrical and dielectric characteristics, may contribute to the development of devices. In particular, manganite achieved vast domain of requests such as magnetic refrigeration $[2,3]$ and spintronic technologies [4]. For that the main challenge is to develop new promoting in the microelectronic industry by improving their dielectric constants.

For instance, most the physical properties of the manganite are interpreted by the double-exchange mechanism which underlies the happening of electron from $\mathrm{Mn}^{3+}$ to $\mathrm{Mn}^{4+}$ ions via the oxygen atom. Importantly, the physical properties of these materials can be controlled by changing the sintering preparation conditions method and either by the type of the metal ion substitutions on La and/or Mn sites [5]. Among the wide literature on manganite,

*Corresponding author: M Baazaoui, Laboratory of Physical Chemistry of Materials, Department of Physics, Faculty of Sciences of Monastir, University of Monastir, 5000, Monastir, Tunisia

Accepted: May 17, 2021; Published: May 19, 2021

Copyright: (C) 2021 Baazaoui M, et al. This is an open-access article distributed under the terms of the Creative Commons Attribution License, which permits unrestricted use, distribution, and reproduction in any medium, provided the original author and source are credited.

Baazaoui M, et al. Int J At Nucl Phys 2021, 6:025

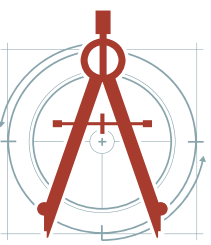


the substitution of metal ion has an important effect which affects the magnetic properties with respect to the electrical conduction properties. These behaviors were generally interpreted from the grains (bulk) and grain boundaries effect [6].

Moreover, studies of the dielectric and electrical properties of materials have been developed in recent years due to their wide field of application. Infact, there are many researches about these properties in different materials type such as $\mathrm{ZnO}$ [7], ZrO2 [8], and the tris (8-hydroxyquinoline) aluminum (Alq3) material [9]. In electronics, the applications of dielectric and ferroelectric materials are in the field of memories. The best known are non-FRAM memories (Ferroelectric Random Access Memory) integrating a ferroelectric material for the storage of information. Perovskite offer interesting advantages for the intended application as high values of electrical permittivity, for example and seem favorable to a "super polarizability" One of the techniques most used for the exploration and the interpretation of the dielectric properties of materials is impedance spectroscopy. It allows in particular detecting the contribution of each microstructure constituent, grain, grain boundaries and effect of the electrodes to the different types of electrical polarization. This method of measuring complex impedance consists in studying the response of a material to the submission to the action of an alternating electric field of variable frequency. In this context; we studied the dielectric properties as a function of the frequency at different temperatures for the sample $\mathrm{Pr}_{0.525} \mathrm{Y}_{0.075} \mathrm{Ca}_{0.1} \mathrm{Sr}_{0.3} \mathrm{Mn}_{0.975} \mathrm{Fe}_{0.025} \mathrm{O}_{3}$ in order to see the effect of temperature on its dielectric properties.

\section{Experimental Details}

The $\mathrm{Pr}_{0.525} \mathrm{Y}_{0.075} \mathrm{Ca}_{0.1} \mathrm{Sr}_{0.3} \mathrm{Mn}_{0.975} \mathrm{Fe}_{0.025} \mathrm{O}_{3}$ sample synthesized by the Sol-gel method. Stoichiometric amounts of $\operatorname{Pr}\left(\mathrm{NO}_{3}\right)_{3} \cdot 6 \mathrm{H}_{2} \mathrm{O}, \mathrm{Y}\left(\mathrm{NO}_{3}\right)_{3} \cdot 6 \mathrm{H}_{2} \mathrm{O}, \mathrm{Ca}\left(\mathrm{NO}_{3}\right)_{2^{\prime}}$ $\mathrm{Sr}\left(\mathrm{NO}_{3}\right)_{2}, \mathrm{Mn}_{2} \mathrm{O}_{3}$ and $\mathrm{Fe}\left(\mathrm{NO}_{3}\right)_{3} \cdot 9 \mathrm{H}_{2} \mathrm{O}$ were dissolved in a distilled water with citric acid. This solution was mixed under regular stirring at $90^{\circ} \mathrm{C}$ in the presence

\section{Pr0.525Y0.075Ca0.1Sr0.3Mn0.975Fe0.025O3}

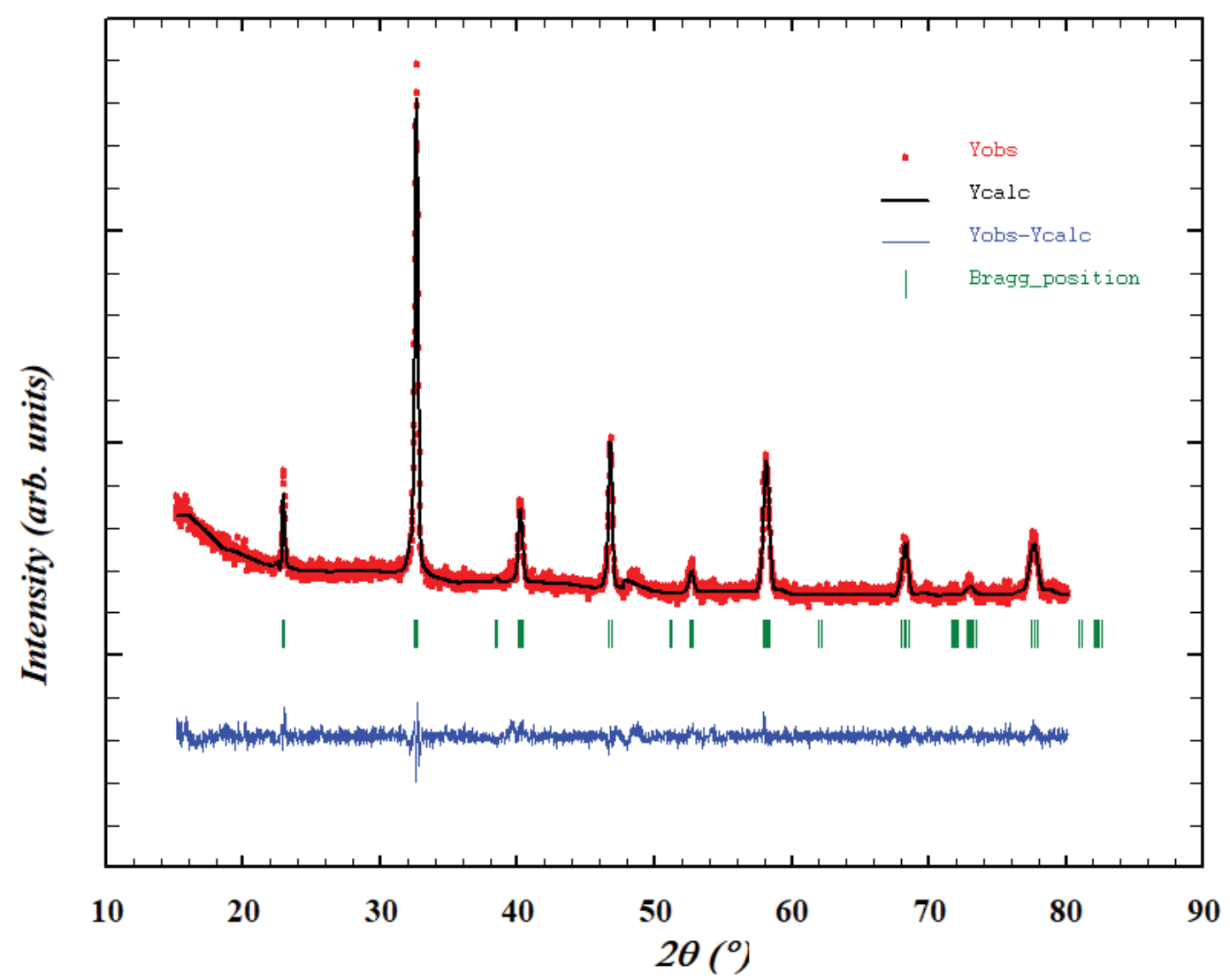

Figure 1: Rietveld refined XRD pattern of $\mathrm{Pr}_{0.525} \mathrm{Y}_{0.075} \mathrm{Ca}_{0.1} \mathrm{Sr}_{0.3} \mathrm{Mn}_{0.975} \mathrm{Fe}_{0.025} \mathrm{O}_{3}$ sample at room temperature. 
Table 1: Refined parameters: Lattice parameters ( $a, b$ and $c)$, cell volume $(v)$, bond length $\left(d_{M n-0}\right)$, bond angle (Mn-O-Mn), obtained from XRD diffraction refinements data of $\mathrm{Pr}_{0.525} \mathrm{Y}_{0.075} \mathrm{Ca}_{0.1} \mathrm{Sr}_{0.3} \mathrm{Mn}_{0.975} \mathrm{Fe}_{0.025} \mathrm{O}_{3}$.

\begin{tabular}{|c|c|}
\hline $\mathbf{x}$ & $\mathrm{Pr}_{0.525} \mathrm{Y}_{0.075} \mathrm{Ca}_{0.1} \mathrm{Sr}_{0.3} \mathrm{Mn}_{0.975} \mathrm{Fe}_{0.025} \mathrm{O}_{3}$ \\
\hline Space group & $R \overline{3} c$ \\
\hline \multicolumn{2}{|l|}{ Lattice parameters } \\
\hline$a=b(\AA ̊)$ & 5.5070 \\
\hline$c(\AA ̊ \AA)$ & 13.4185 \\
\hline$V\left(\AA^{3}\right)$ & 352.422 \\
\hline $\mathrm{Pr} / \mathrm{Y} / \mathrm{Ca} / \mathrm{Sr} \mathrm{B}_{\text {iso }}\left(\AA^{2}\right)$ & 1.223 \\
\hline $\mathrm{Mn} / \mathrm{Fe} \mathrm{B}_{\text {iso }}\left(\AA^{2}\right)$ & 0.526 \\
\hline$O B_{\text {iso }}\left(\AA^{2}\right)$ & 1.163 \\
\hline$x(0)$ & 0.546 \\
\hline$\left.d_{M n-0(} \AA\right)$ & 1.96 \\
\hline$\vartheta_{(M n-O-M n)}\left({ }^{\circ}\right)$ & 165.15 \\
\hline$R_{w p}$ & 5.76 \\
\hline$R_{p}$ & 4.58 \\
\hline$R_{\exp }$ & 5.11 \\
\hline$\chi^{2}$ & 1.27 \\
\hline
\end{tabular}

Table 2: The fitting parameters obtained from the experimental data of conductance as a function of frequency.

\begin{tabular}{|l|l|l|l|}
\hline $\mathbf{T}(\mathbf{K})$ & $\mathbf{n}$ & $\mathbf{G}_{\mathbf{D C}}(\mathbf{S})$ & $\mathbf{R}^{\mathbf{2}}$ \\
\hline 295 & 1.147 & $3.32 .10^{-7}$ & 0.998 \\
\hline 320 & 1.194 & $4.22 .10^{-7}$ & 0.999 \\
\hline 340 & 1.20 & $7.45 .10^{-7}$ & 0.999 \\
\hline 360 & 1.12 & $1.15 .10^{-6}$ & 0.999 \\
\hline 380 & 1.076 & $1.41 .10^{-6}$ & 0.999 \\
\hline 400 & 1.08 & $3.29 .10^{-6}$ & 0.999 \\
\hline 420 & 1.104 & $4.84 .10^{-6}$ & 0.998 \\
\hline
\end{tabular}

of ethylene glycol to obtain a homogeneous gel. Then, the obtained gel was calcined at $500 \mathrm{~K}$ for $8 \mathrm{~h}$ to eliminate the organic material. After that, the obtained powders were pelletized into pellets with diameter $13 \mathrm{~mm}$ and thickness of $1 \mathrm{~mm}$ under a pressure of 4 tons $/ \mathrm{cm}^{2}$. Finally, the pellets were sintered at $1000{ }^{\circ} \mathrm{C}$ for $24 \mathrm{~h}$. The structural characterization carried out by X-ray diffraction using an automatic two-circle diffractometer. Analysis of the X-ray diffractograms obtained at room temperature showed that the lines are intense and fine, proof of good crystallization of the compounds. The $\mathrm{X}$-ray diffraction spectra of sample $\operatorname{Pr}_{0.525} \mathrm{Y}_{0.075} \mathrm{Ca}_{0.1} \mathrm{Sr}_{0.3} \mathrm{Mn}_{0.975} \mathrm{Fe}_{0.025} \mathrm{O}_{3}$ are refined by the Rietveld method using Full prof software. It was found that the compound crystallizes in the rhombohedra structure with the symmetry of $R \overline{3} c$ (see Figure 1 and Table 1). In addition, the electrical measurements were carried out on PSM1735 model impedance spectroscopy in the temperature varying between 295 and $420 \mathrm{~K}$.

\section{Electrical Conductivity Measurement}

The study of electrical conductance is one of the most significant characteristics of manganite which describes the phenomena of charge carrier's hopping. Figure 2 shows the variation of conductance versus frequency recorded in the temperature range [295-420] $\mathrm{K}$ for our sample. The increase of conductance is clear with increasing frequency. Importantly, the conductance curve present two regions part. The first one is located at a low frequency region described by the presence of a Plateau region independent of frequency; Named $\sigma_{d c}$. Additionally, the second region; named $\sigma_{\mathrm{ac}}$ is located at a high frequency. It is noteworthy that the total conductance is the sum of $\sigma_{\mathrm{dc}}$ and $\sigma_{\mathrm{ac}}$ explained by the "universal" Jonscher power law [10] as follow:

$$
G(\omega)=G^{d c}+G^{a c}=G^{d c}+A \omega^{n}
$$

Where $A$ is a constant describing the strength of polarizability and $\mathrm{n}$ is the power exponent with $0 \leq$ $\mathrm{n} \leq 1$.

Using Equation 1, the experimental conductivity data were well fitted as shown in Figure 3. It is clear that the fit curve coincidences well with the experimental values. It can be seen from the fitted result presented in Table 2 that the achieved $\mathrm{n}$ values are all superior than 1 for the whole temperature range. This behavior indicates that there are hops between neighboring sites [11].

\section{Conductance Analysis}

Figure 4 displays the temperature dependence $G_{D C}$ conductance measurement in temperature range 295-420 $\mathrm{K}$ for $\operatorname{Pr}_{0.525} \mathrm{Y}_{0}$. ${ }_{075} \mathrm{Ca}_{0.1} \mathrm{Sr}_{0.3} \mathrm{Mn}_{0.975} \mathrm{Fe}_{0.025} \mathrm{O}_{3}$ sample. Throughout 


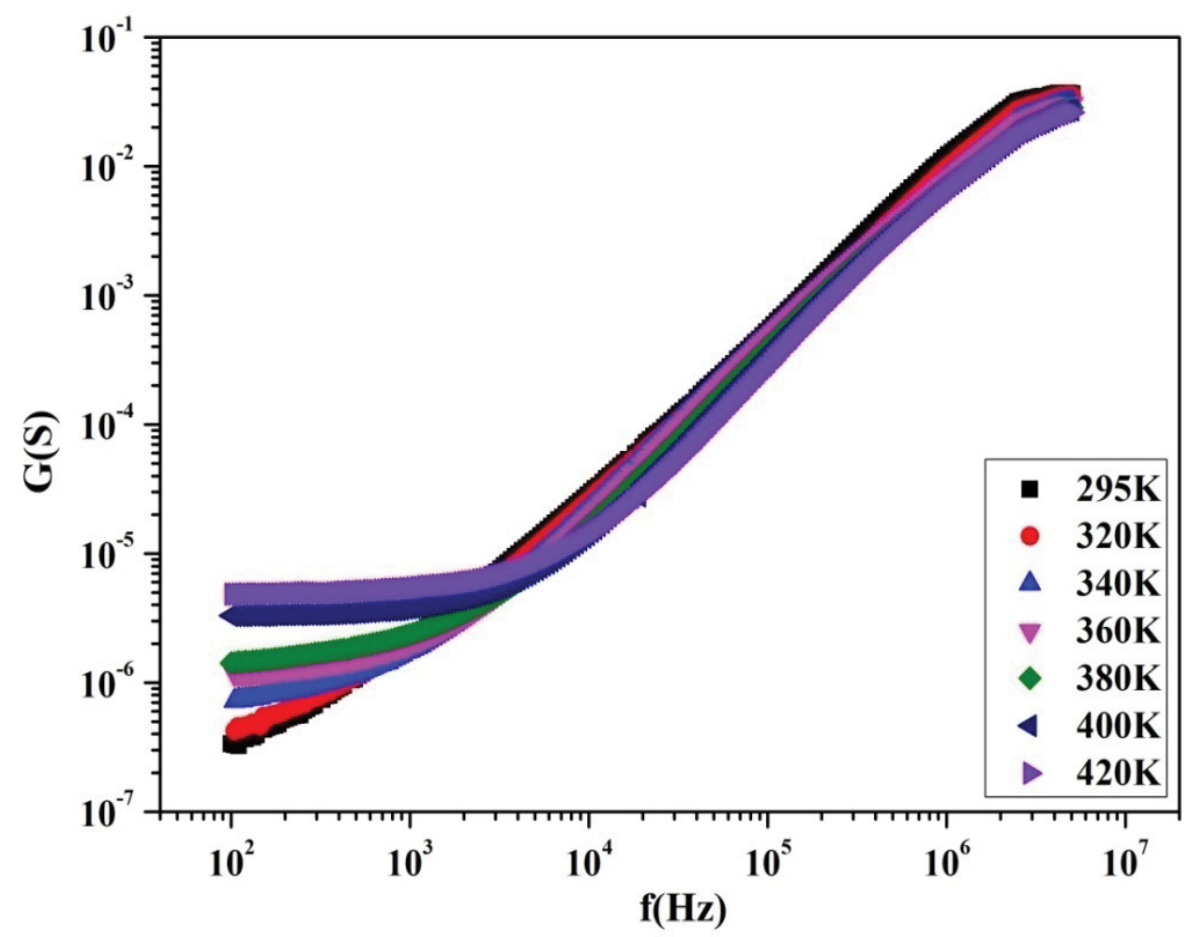

Figure 2: Variation of the conductance with frequency at different temperatures.

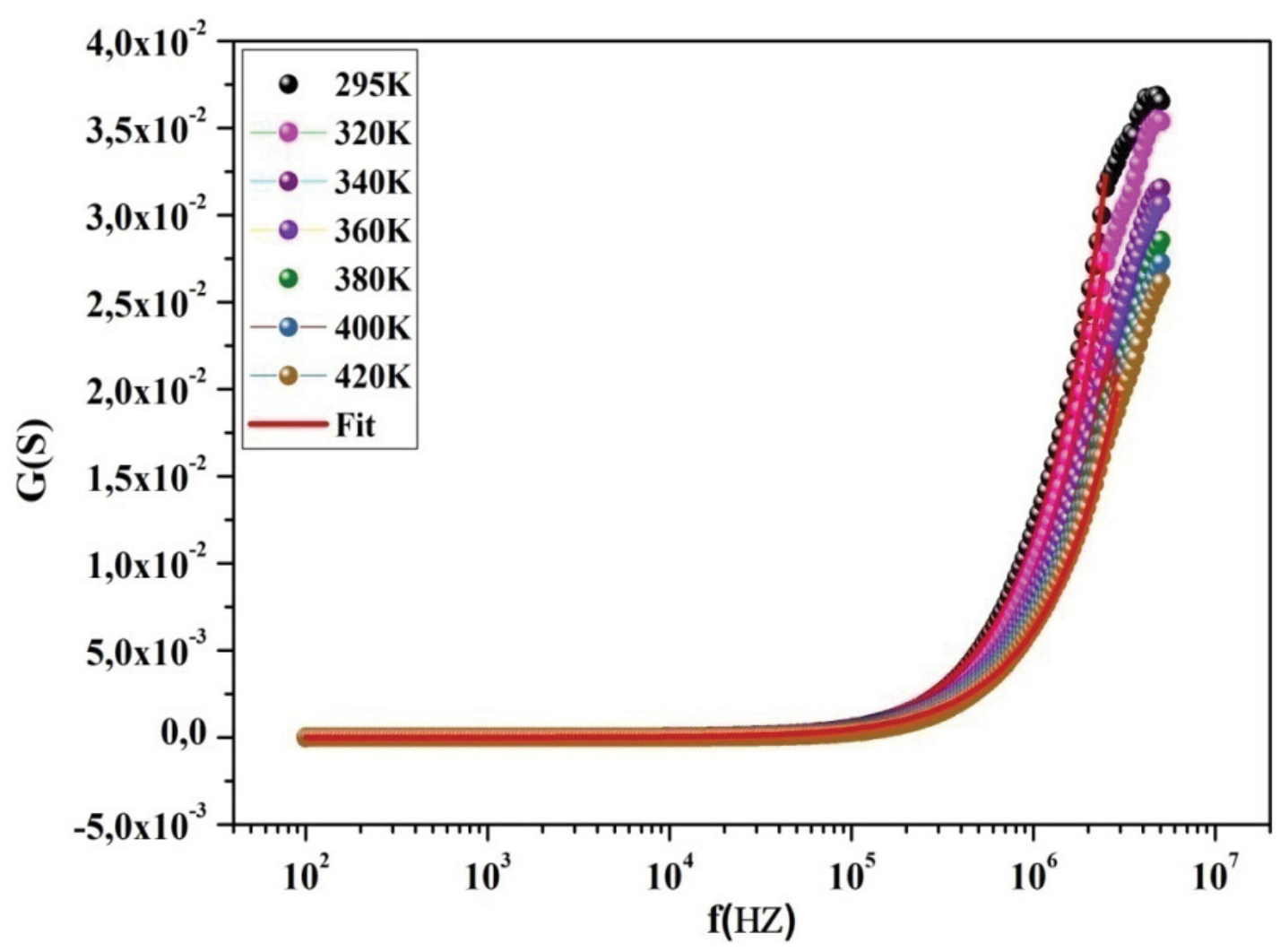

Figure 3: Fitting of the conductance $\mathrm{G}$ at different temperature. 


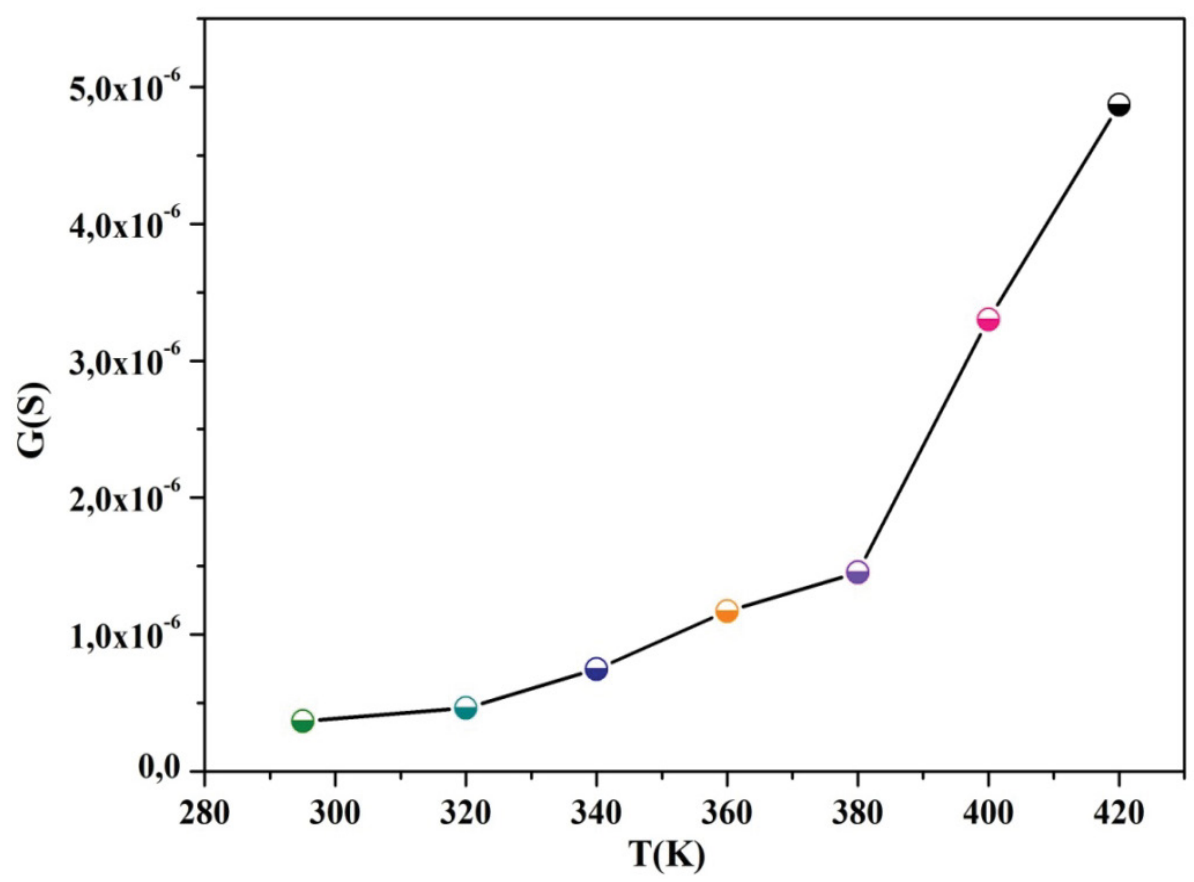

Figure 4: Temperature dependence of $G_{D C}$ conductance measurement. The inset show the variation of $\operatorname{Ln}(G T)$ as a function of $(1000 / T)$ in which the red solid line is the linear fit of our experimental data.

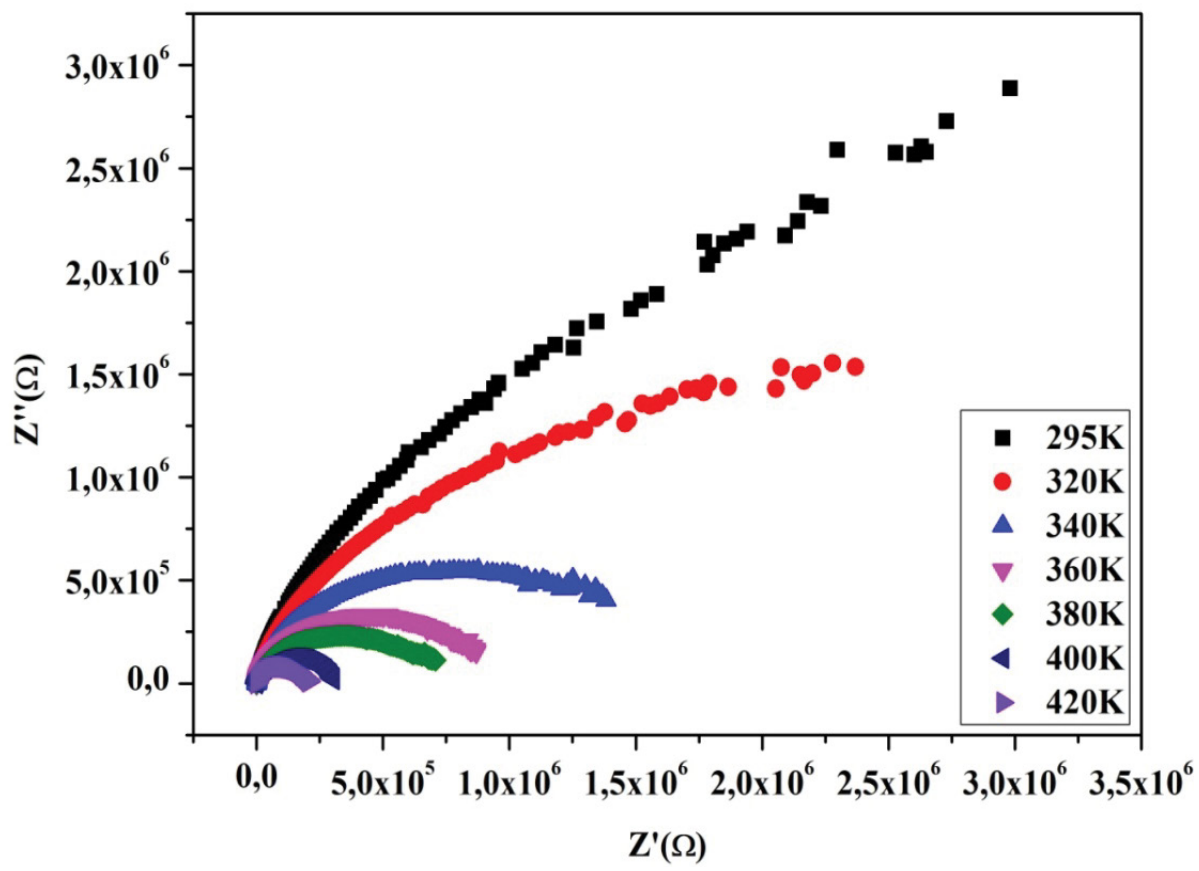

Figure 5: Nyquist plots (-Z" vs. Z') of impedance data at different temperatures.

the temperature range, we observe the increase of the conductance as function of temperature which proves only a semiconductor behavior in our compounds.

In our case the experimental data of the conductance were well fitted by the Mott and Davis law given by:

$$
G_{D C}=G_{0} \exp \left(\frac{-E_{a}}{K_{B} T}\right)
$$

Where $G_{0}$ is a pre-exponential factor, $E_{a}$ is the activation energy and $\mathrm{k}_{\mathrm{B}}$ is the Boltzmann constant. 
From the linear fit of the experimental data as shown in the inset of Figure 4, the determined activation energy is found to be $E_{a}=0.117 \mathrm{ev}$.

\section{Impedance Analysis}

For our composition the impedance analysis is carried out in order to investigate the microstructure contributions like grains and grain boundaries with the relaxation process. For that we plot the Nyquist diagram which is defined as the plot between real part of the impedance $\left(Z^{\prime}\right)$ and imaginary part of the impedance $\left(Z^{\prime \prime}\right)$, in the temperature range 295$420 \mathrm{~K}$ as shown in Figure 5. It is clearly from this diagram the appearance of semicircular arcs which is characterized by the decreasing of their diameter with increasing temperature. From the achieved single semicircle, we can conclude that the electrical mechanism for each temperature indicate a nonDebye type relaxation behavior [12]. This, decrease is related to the increase of dc conduction. Further, to determine the corresponding resistance $(R)$ and capacitance $(C)$ values we have employed an equivalent circuit model by fitting the Nyquist diagram for all temperature. The fitted results are shown by solid lines in which the best fit is composed with a three parallel $R C$ circuits combined in series as shown in Figure 6. The fitting results are grouped illustrated in Table 3 for each temperature.

From the results fit we can deduce that the

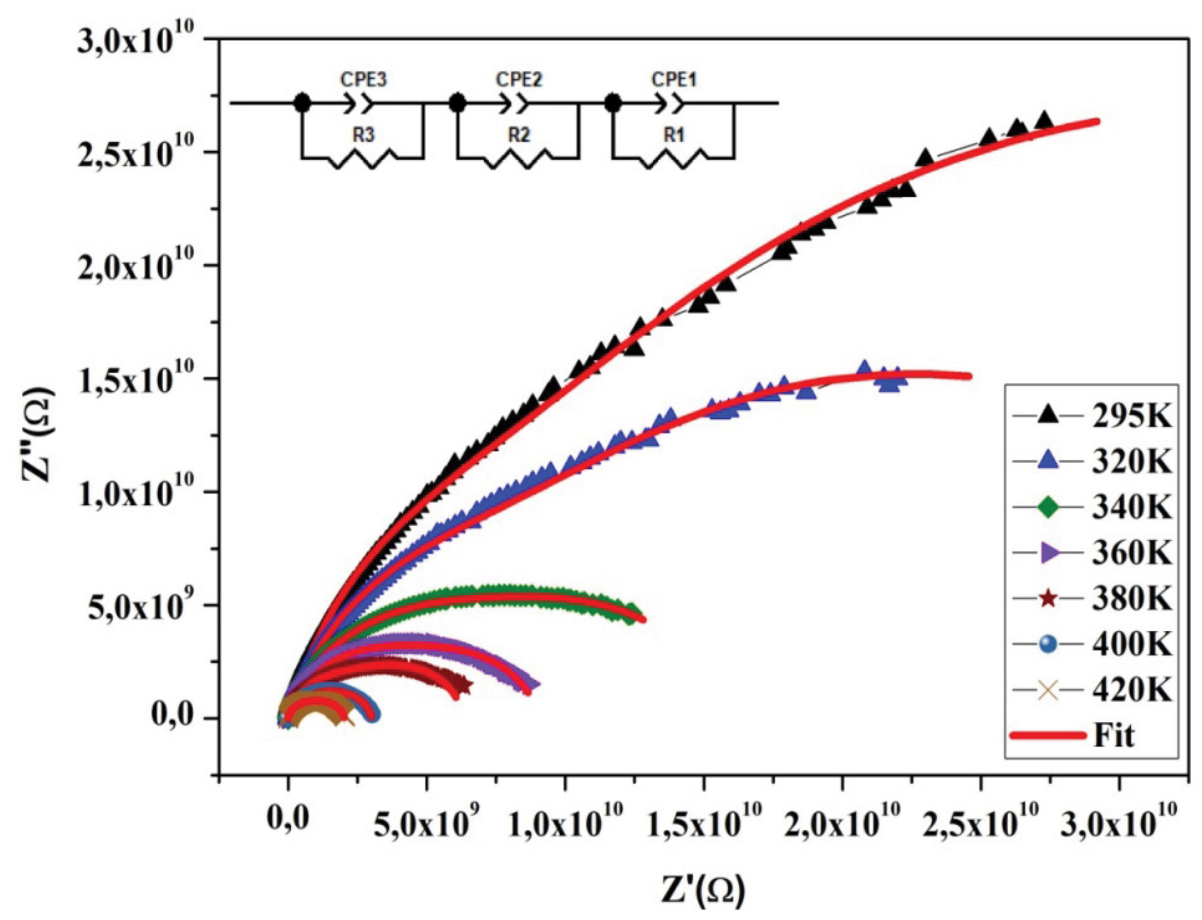

Figure 6: Fitting of the nyquist plots at different temperatures.

Table 3: Electrical parameters of the equivalent circuit deduced from complex impedance spectrum for different temperatures.

\begin{tabular}{|l|l|l|l|l|}
\hline $\mathbf{T}(\mathbf{K})$ & $\mathbf{R g}\left(\mathbf{\Omega}^{-\mathbf{1}}\right)$ & $\mathbf{C g}(\mathbf{F})^{*} \mathbf{1 0}^{-\mathbf{1 0}}$ & $\mathbf{R g b}\left(\mathbf{\Omega}^{-\mathbf{1}}\right)$ & $\mathbf{C g b}(\mathbf{F})^{*} \mathbf{1 0}^{-\mathbf{1 0}}$ \\
\hline 295 & 954280 & 2.908 & $5.4109^{*} 10^{6}$ & 3.964 \\
\hline 320 & 847790 & 2.752 & $3.006^{*} 10^{6}$ & 6.082 \\
\hline 340 & 683210 & 2.295 & 802030 & 0.1081 \\
\hline 360 & 475520 & 0.1105 & 529100 & 1.024 \\
\hline 380 & 300100 & 5.082 & 325330 & 2.378 \\
\hline 400 & 99980 & 5.299 & 200700 & 2.440 \\
\hline 420 & 87714 & 8.036 & 114950 & 2.228 \\
\hline
\end{tabular}

T: Temperature; Rg: Grain resistance; Rgb: Grain-boundary resistance; Cgb: Grain-boundary capacitance. 
Nyquist plot is due to the superposition of three contributions. In other word three distinct semicircular arcs are not observed; one corresponds to the grain resistance contribution located at high frequency RC element; the medium corresponds to grain boundary resistance and the low is associated to electrode contributions [13].

The grain-boundary resistance $R_{g b}$ appears to be larger than the grain resistance $R_{g b}$. Those assigned to the disordered atomic arrangement located near the grain boundary region which assure a significant increase in electron scattering.

Interestingly, we remark the decrease of the $R_{g b}$ values with the increase of temperature. Thus, this decrease suggests semiconductor behavior for this sample [14]. Additionally this behavior can be interpreted by the lowering of barrier which lead to a significant increase in the concentration of mobile charges and then enhance the electrical transport as rising temperature [15].

\section{Impedance Analysis}

Figure 7 presents the variation of real part of impedance $\left(Z^{\prime}\right)$ as a function with the frequency at various temperatures range. It is determined that $Z^{\prime}$ value decreases as increasing frequency whose merges for all the temperatures in the high frequency range. This behavior suggests the presence of the space charge polarization [16].
We also note in lower frequency region, that the $Z^{\prime}$ value decreases with increasing temperature, indicating reduction of barrier potential which is responsible for the enhancement of conductivity $[17,18]$.

In Figure 8 we present the frequency dependence of the imaginary part $Z$ " of the impedance in the temperature region $295-420 \mathrm{~K}$.

The higher peak of $Z^{\prime \prime}$ decreases gradually, with increasing temperature and its broadening increases. This variation indicates the existence of immobile species in our compounds. Further, at the high-frequency range, the merging in all the $Z^{\prime \prime}$ plots can be explained by the accumulation of space charge. It should also be mentioned the appearance of a maximum peak $Z^{\prime \prime}{ }_{\text {max }}$ at particular relaxation frequency as increasing temperature. This peak shift towards higher frequencies as temperature increases. This behavior is related to the polarization phenomena due to the contribution the mobility of the charge carriers on the relaxation frequency which correspond to the thermally activated relaxation [19]. Additionally, the coincidence of the $Z$ " curves at high frequencies region approves the liberation of charge at the grain.

\section{Dielectric Permittivity}

The complex permittivity $\varepsilon^{*}(\omega)$ is known as $\varepsilon^{*}(\omega)=\varepsilon^{\prime}(\omega)-j \varepsilon^{\prime \prime}(\omega)[20]$, in which the real part

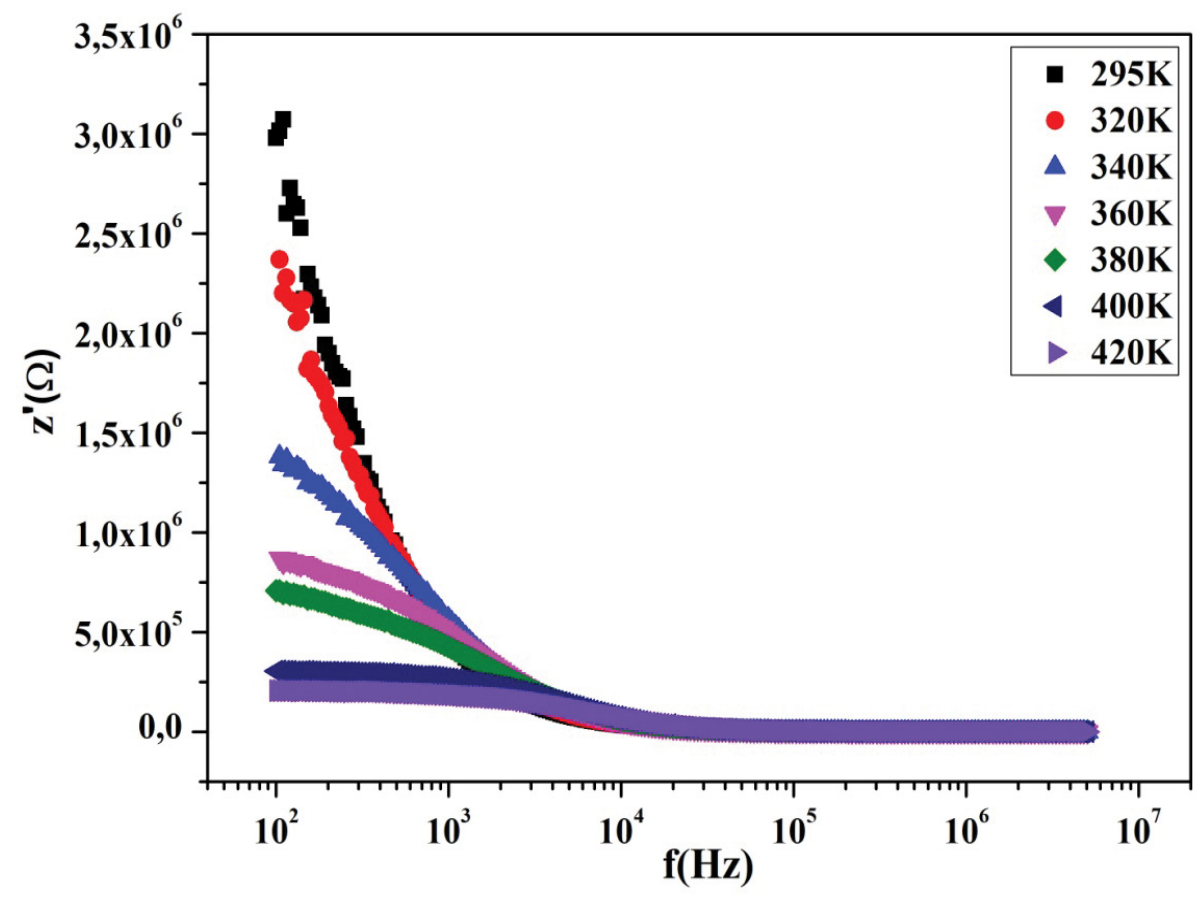

Figure 7: Variation of real Z' part of impedance with frequency at different temperatures. 


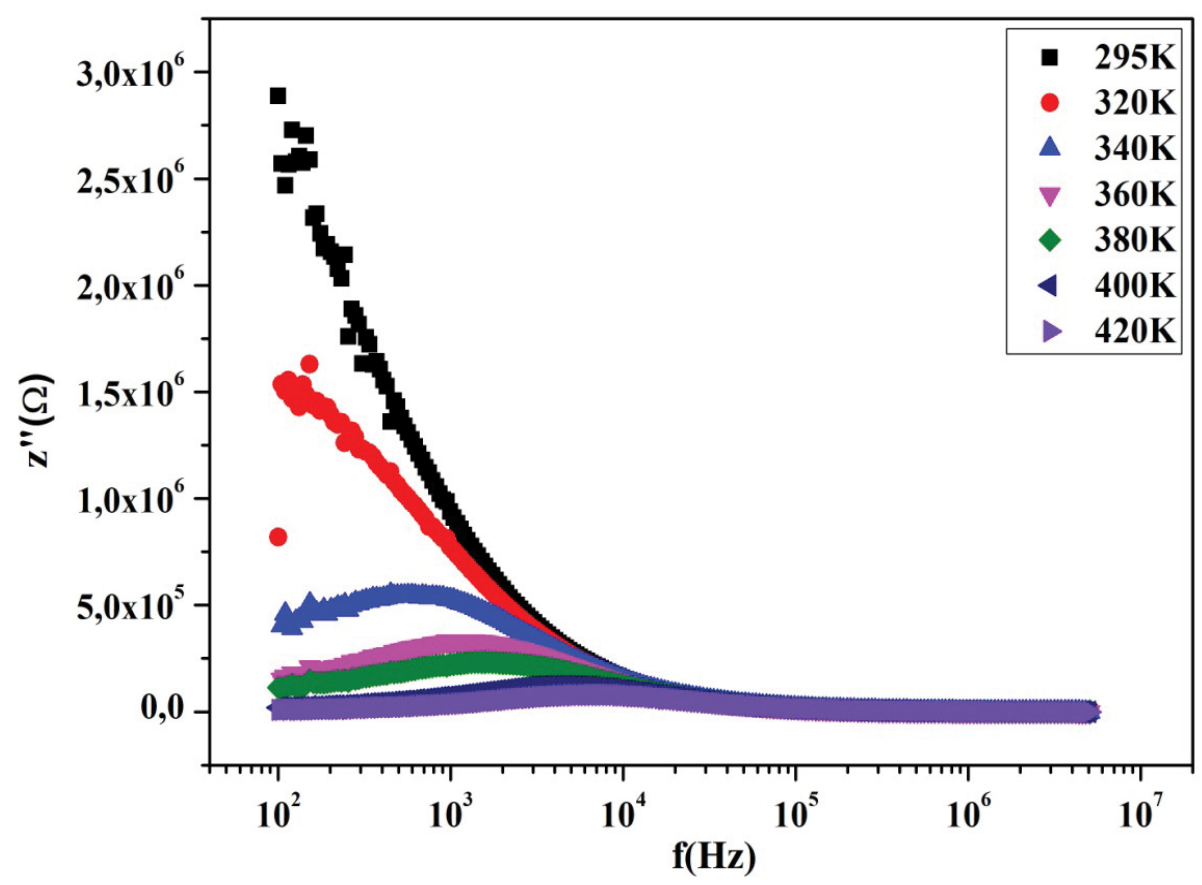

Figure 8: Variation of Imaginary Z" part of impedance with frequency at different temperatures.

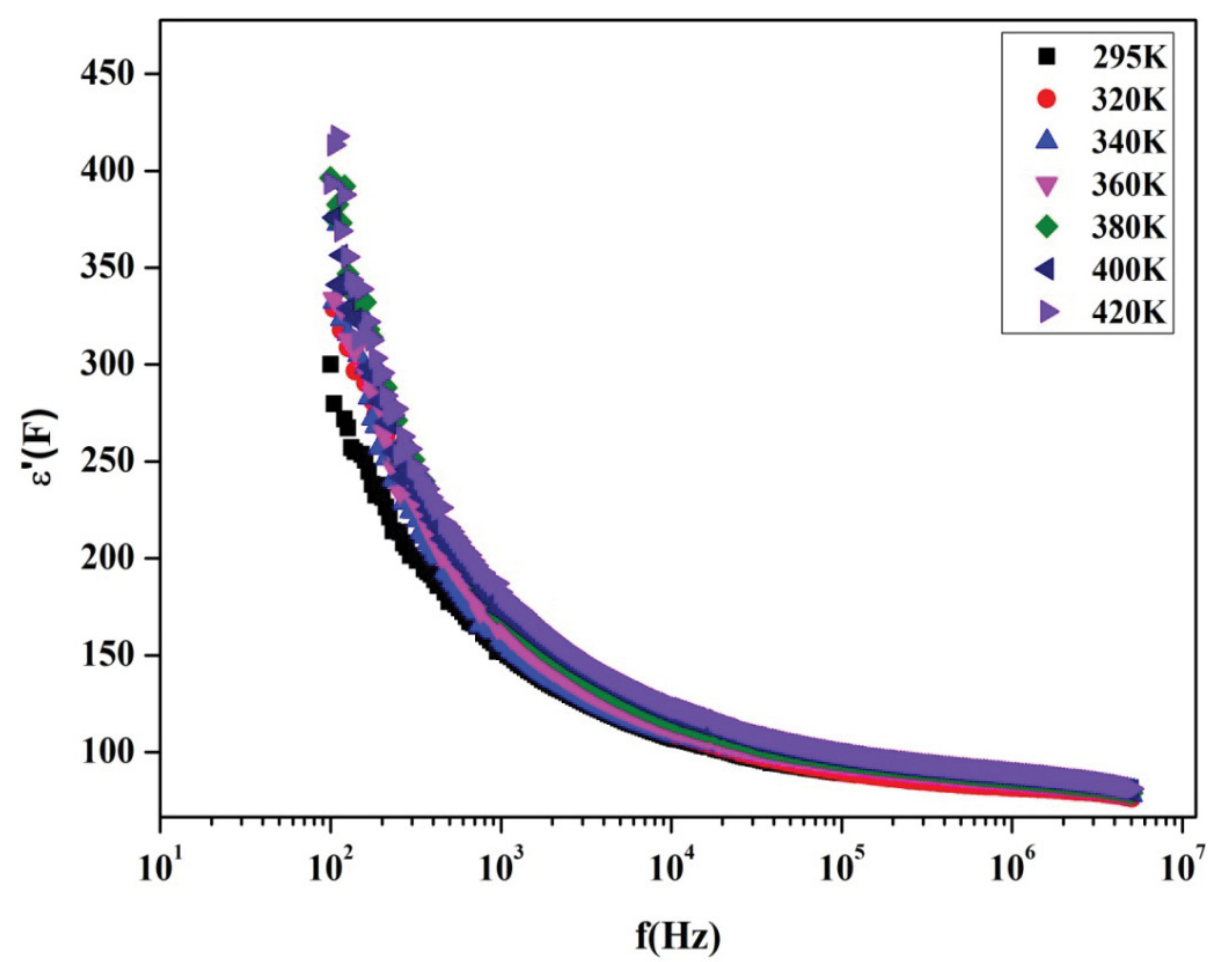

Figure 9: Frequency dependence of the real part of dielectric permittivity constant at different temperature.

$\varepsilon^{\prime}$ describes the stored energy and the imaginary one $\varepsilon^{\prime \prime}$ gives a detail of the dissipation energy, respectively. These values are achieved from the following expression: [21]

$$
\varepsilon^{*}=\frac{1}{j w C 0 Z^{*}}
$$

Where the geometric capacitance $C_{0}=\varepsilon_{0} S / e . \varepsilon_{0}$ is the free space permittivity and $S$ and e are the electrodes area and the thickness of the sample, respectively.

In Figure 9 we display the frequency dependence of the permittivity $\varepsilon^{\prime}$ in the temperatures ranges 
295-420 K for sample. It is clear at low frequencies that $\varepsilon^{\prime}$ reach a large value which decreases abruptly until the coincidence of $\varepsilon^{\prime}$ values with all temperatures at high frequencies.

This behavior suggests a change in the dielectric evolution in our compounds [22]. In fact, the dipoles cannot respect the orientation of the electric field as the frequency increases which assure the strongly decrease of the permittivity. In our case the increase in temperature contribute to disorder the dipoles which induces the decrease of $\varepsilon^{\prime}$ values [23].

In addition, we studied the dielectric loss factor $\tan \delta$ which is given as " $\tan \delta=\varepsilon^{\prime \prime} / \varepsilon^{\prime}$. Figure 10 displays the $\tan \delta$ variation versus frequency in the temperature range $295-420 \mathrm{~K}$. An abrupt decrease of $\tan \delta$ is clearly observed from the curves with the increase of frequency assuming the usual dielectric dispersion. It is well known that when the resistivity value is high, at low frequencies, the grain boundary contribution is dominant due to the contribution from the interfacial polarization [24]. For that we need more electrical energy to assure the mobility of charges carriers. On the other hand, the abrupt decrease of $\tan \delta$ with frequency is related to the decrease of the grain resistance at high frequencies. Nevertheless, $\tan \delta$ increase with the temperature increase. This behavior confirms the thermal activation of the dielectric relaxation [24].

\section{Complex Modulus}

Based on the complex modulus measurements we can separate the grain from the grain boundary contribution without any intervention of the electrode response.

The complex electrical modulus is defined as: $M^{*}$ $=M^{\prime}+j M^{\prime \prime}$, which can be expressed as:

$$
M=\frac{\varepsilon}{\varepsilon^{2}+\varepsilon^{\prime \prime 2}} \text { and } M=\frac{\varepsilon^{\prime \prime}}{\varepsilon^{2}+\varepsilon^{\prime 2}}
$$

In Figure 11 we present the frequency dependence evolution of the real part $M^{\prime}$ at different temperatures. At low frequency it is evident that $\mathrm{M}^{\prime}$ values reach zero. This behavior can be interpreted in terms of long-range mobility of charge which consequently indicates the negligible contribution of electrode influence [25]. However, at higher frequency range we detect a continuous dispersion evolution which indicates the occurrence of shortrange mobility charge conduction [26].

Figure 12 shows the evolution of the imaginary part of the modulus versus of frequency for different temperature. In our case we can see the shift of the maximum peak to higher frequency as

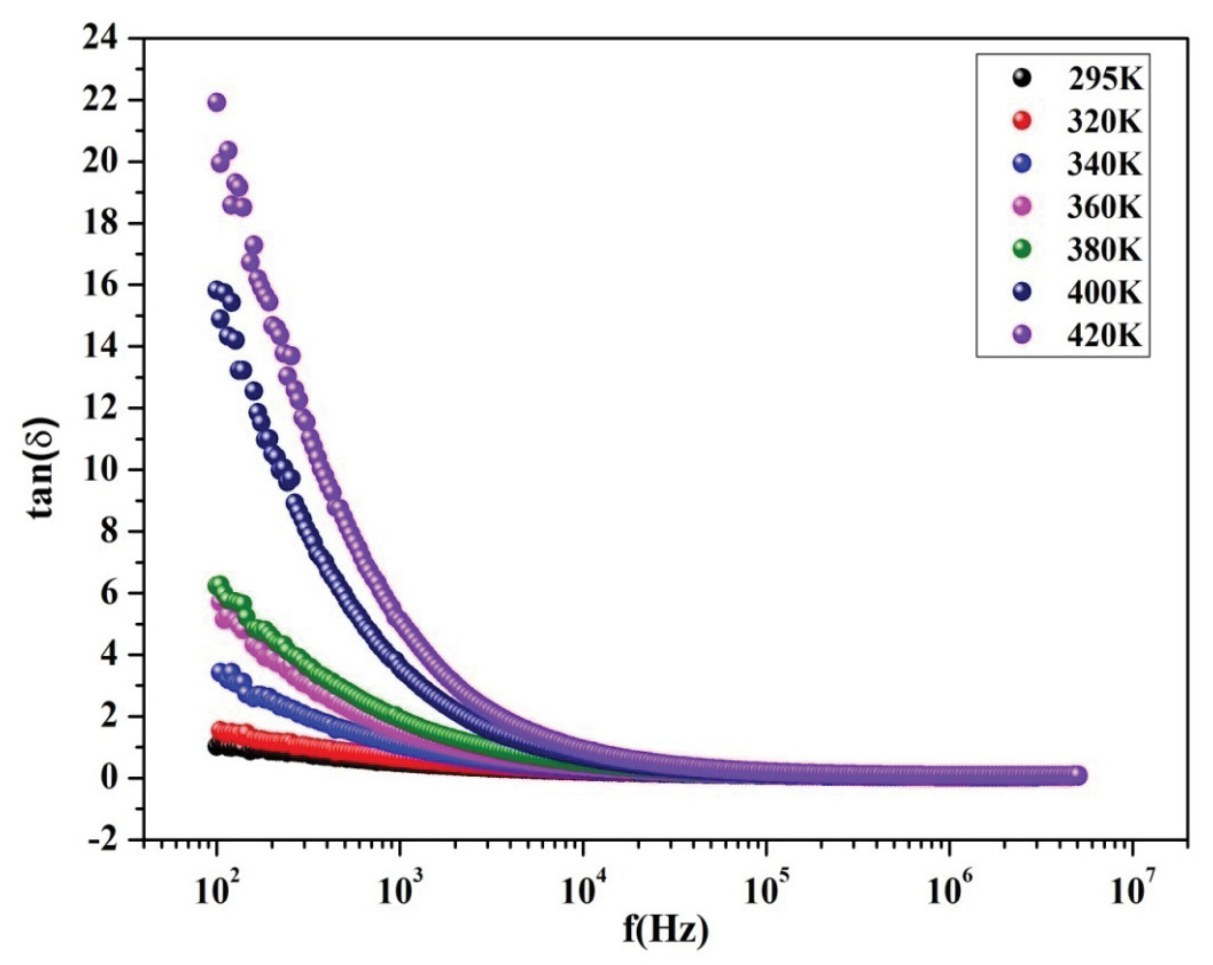

Figure 10: Dielectric loss factor as function of frequency for different temperature. 


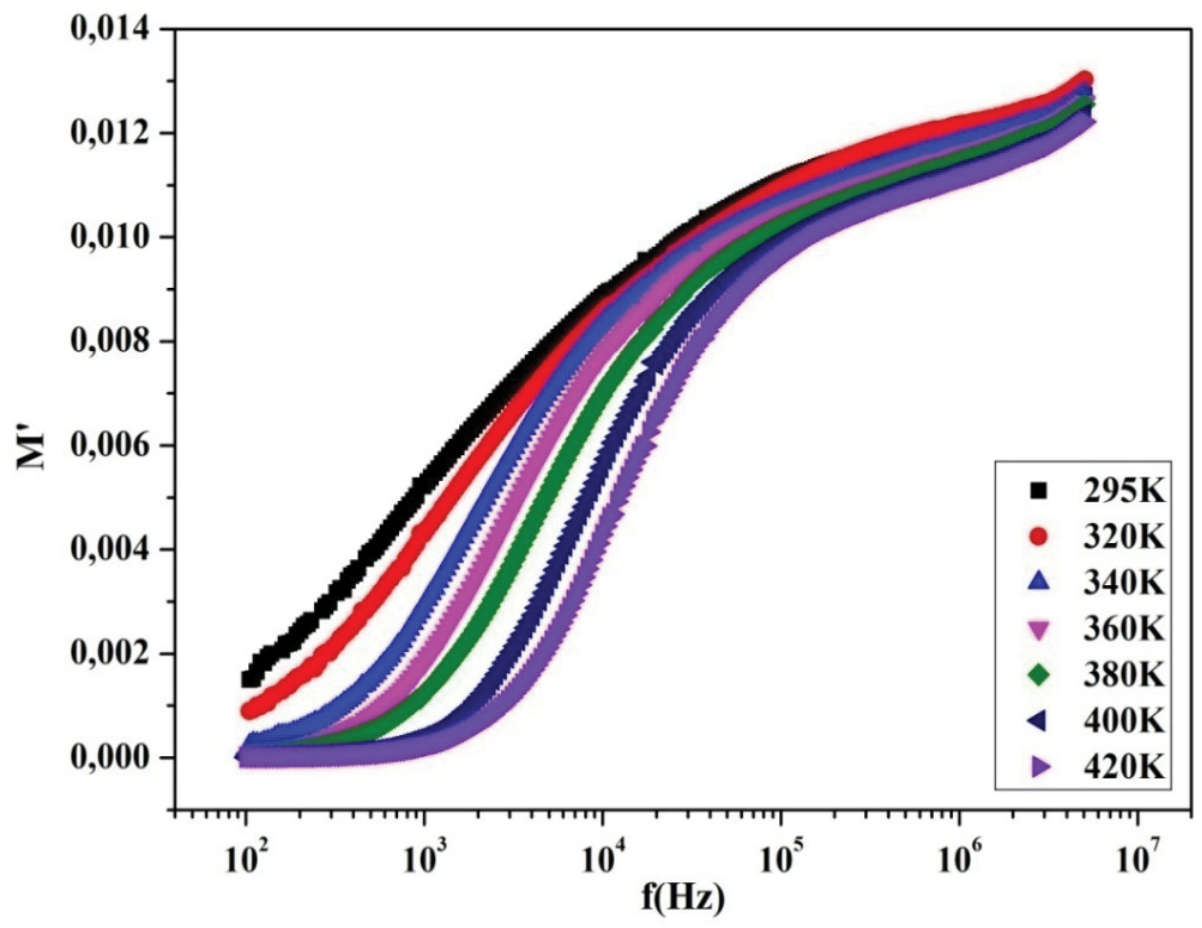

Figure 11: Variation of real modulus part as function of frequency for different temperature.

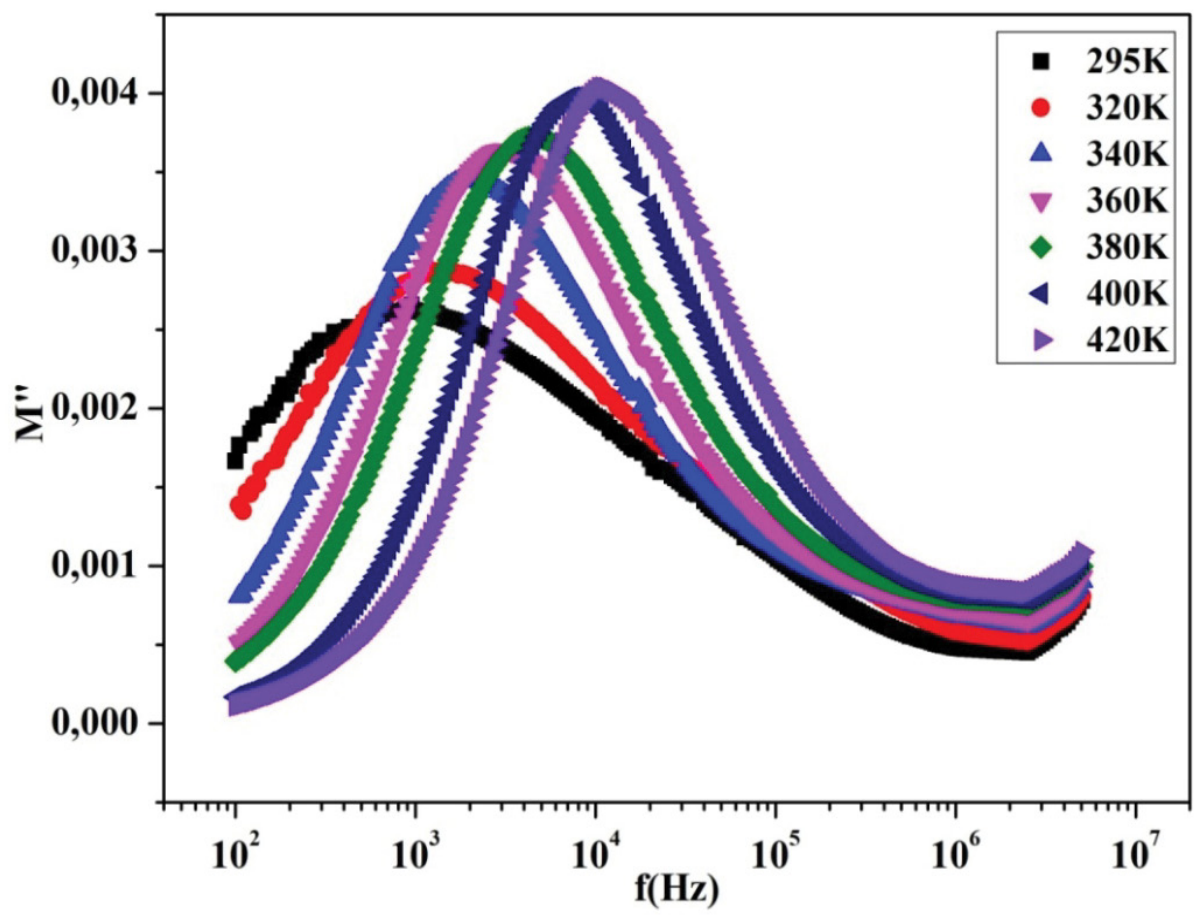

Figure 12: Variation of imaginary modulus part as function of frequency for different temperature.

increasing temperature. This behavior suggests the dependence of relaxation phenomena with temperature in our sample. Additionally, this peak is associated with the grain boundary response which indicates that the relaxation time for this mechanism decreases with temperature [27].

\section{Conclusion}

The structural and dielectric properties were investigated using complex impedance spectroscopy versus frequency and temperature for the system $\operatorname{Pr}_{0.525} \mathrm{Y}_{0.075} \mathrm{Ca}_{0.1} \mathrm{Sr}_{0.3} \mathrm{Mn}_{0.975} \mathrm{Fe}_{0.025} \mathrm{O}_{3}$. 
Powder XRD patterns revealed the crystallization of our sample in a rhombohedral structure $(R \overline{3} c$ space group). The increase of the conductance as function of temperature confirms the semiconductor behavior in our system. The complex impedance spectra revealed the appearance of semicircle arcs, well modeled in terms of electrical equivalent circuit. Additionally, the decrease of the diameter of semicircular arcs with increasing temperature confirms the non-Debye type relaxation behavior in our compound.

\section{References}

1. Baazaoui M, Hamdaoui N, Laouyenne MR, Cheikhrouhou W, Beji L, et al. (2017) Role of gallium doping in structural, magnetic, and dielectric properties of the compounds $\mathrm{La}_{0.65} \mathrm{Bi}_{0.05} \mathrm{Sr}_{0.3} \mathrm{Mn}_{1-}$ $\mathrm{Ga}_{x} \mathrm{O}_{3}(x=0$ and 0.06). J Super cond Nov Magn 30: 2763-2770.

2. Brown JS, Domanski PA (2014) Review of alternative cooling technologies. Appl Therm Eng 64: 252-262.

3. Hassayoun O, Baazaoui M, Laouyenne MR, Hosni F, Hlil EK, et al. (2019) Magnetocaloric effect and electron paramagnetic resonance studies of the transition from ferromagnetic to paramagnetic in $\mathrm{La}_{0.8} \mathrm{Na}_{0.2} \mathrm{Mn}_{1-\mathrm{x}} \mathrm{Ni}_{\mathrm{x}} \mathrm{O}_{3}(0 \leq \mathrm{x} \leq 0.06)$. J Physics and Chemistry of Solids 135: 109058.

4. Lisauskas A, Khartsev SI, Grishin A (2000) Tailoring the colossal magnetoresistivity: La0.7(Pb0.63Sr0.37)0.3 $\mathrm{MnO}_{3}$ thin-film uncooled bolometer. Appl Phys Lett 77: 756-758.

5. Laouyenne MR, Baazaoui M, Mahjoub SA, Cheikhrouhou-Koubaa W, Oumezzine M (2017) Enhanced magnetocaloric effect with the high tunability of bismuth in $\mathrm{La}_{0.8} \mathrm{Na}_{0.2} \mathrm{Mn}_{1-\mathrm{x}} \mathrm{Bi}_{\mathrm{x}} \mathrm{O}_{3}(0 \leq \mathrm{x} \leq$ $0.06)$ perovskite manganites. J Alloys Compd 720 : 212-220.

6. Peng WY, Zhang JH (2006) Magnetostriction studies in an antiferromagnetic polycrystalline Mn42Fe58 alloy. Appl Phys Lett 89: 262501.

7. Thabit HA, Kabir NA, Ahmed NM, Alraddadi S, Al Buriahi MS (2021) Synthesis, structural, optical, and thermoluminescence properties of $\mathrm{ZnO} / \mathrm{Ag} / \mathrm{Y}$ nano powders for electronic and dosimetry applications. Ceramics International 47: 4249-4256.

8. Al Buriahi MS, Alomairy S, Saeed A, Abouhaswa AS, Rammah YS (2021) Effect of ZrO2 addition on electrical and mechanical properties of B2O3-PbOLi203 glasses. Ceramics International 47: 1306513070.
9. Saeed A, Al-Buriahi MS, Razvi MAN, Salah N, AlHazmi FE (2021) Electrical and dielectric properties of meridional and facial $\mathrm{Alq}_{3}$ nanorods powders. Journal of Materials Science: Materials in Electronics 32: 2075-2087.

10.Firloaga PS, Poienar M, Malaescu I, Lungu A, Mihali CV, et al. (2018) Electrical conductivity of Ca-substituted lanthanum manganites. Ceramics International 44: 5823-5828.

11. Hsini M, Hamdaoui N, Hcini S (2017) Effect of iron doping at $\mathrm{Mn}$-site on complex impedance spectroscopy properties of $\mathrm{Nd} 0.67$ Ba $0.33 \mathrm{MnO} 3$ perovskite. Phase Transitions 91: 1-16.

12.Singh NK (2012) Microstructure and dielectric relaxation of $\mathrm{BT}$ and $\mathrm{ST}$ doped $\mathrm{Ba}(\mathrm{Fe} 0.5 \mathrm{Nb0.5}) \mathrm{O} 3$ ceramics for sensor applications. Adv Mat Lett 3: 181-187.

13. Mohan U, Gogoi P, Baruah SK (2016) Impedance Spectroscopy Study of the AC Conductivity and Dielectric properties of 3, 5-Dimethylpyridinelodine Charge Transfer Complex. Oriental Journal of Chemistry 32: 1003-1014.

14. Khadhraoui S, Triki A, Hcini S, Zemnia S, Oumezzine M (2014) Variable-range-hopping conduction and dielectric relaxation in Pr0.6Sr0.4Mn0.6Ti0.4O3 $\S \mathrm{d}$ perovskite. J Magn Magn Mater 371: 69-76.

15. Pradhan DK, Samantary BK, Chaudhary RNP, Thakur AK (2005) Complex impedance studies on a layered perovskite ceramic oxide - NaNdTiO4. Mater Sci Eng B 116: 7-13.

16.Rahmouni H, Smari M, Cherif B, Dhahri E, Khirouni $\mathrm{K}$ (2015) Conduction mechanism, impedance spectroscopic investigation and dielectric behavior of La0.5Ca0.5-xAgxMnO3 manganites with compositions below the concentration limit of silver solubility in perovskites $(0 \leq \mathrm{x} \leq 0.2)$. J Dalto Transc 44: 10457-10466.

17. Costa MM, Pires Jr GFM, Sombra ASB (2010) Dielectric and impedance properties' studies of the of lead doped (PbO)-Co2Y type hexaferrite (Ba2Co2Fe12O22 (Co2Y)). Mat Chem Phys 123: 35-39.

18.Ahmad Z, Atiq S, Abbas SK, Ramay SM, Riaz S, et al. (2016) Structural and complex impedance spectroscopic studies of Mg-substituted CoFe2O4. Ceramics International 42: 18271-18282.

19. Kumar M, Shankar S, Brijmohan, Kumar S, Thakur OP, et al. (2017) Impedance spectroscopy and conductivity analysis of multiferroic BFO-BT solid solutions Physics Letters A 381: 379-386. 
20.Cole KS, Cole RH (1941) Dispersion and absorption in dielectrics - I. Alternating current characteristics. J Chem Phys 9: 341.

21.von Hippel A (1954) Dielectrics and waves. John Wiley and Sons, New York, USA.

22.Hamdaoui N, Azizian-Kalandaragh Y, Khlifi M, Beji L (2019) Structural, magnetic and dielectric properties of Ni0.6Mg0.4Fe2O4 ferromagnetic ferrite prepared by sol gel method. Ceramics International 45: 1645816465.

23.Ben Jazia Kharrat A, Moussa S, Moutiaa N, Khirouni K, Boujelben W (2017) Structural, electrical and dielectric properties of Bi-doped Pr0.8xBixSr0.2MnO3 manganite oxides prepared by solgel process. Journal of Alloys and Compounds 724: 389-399.

24.Sun Z, Li L, Yu S, Kang X, Chen S (2017) Energy storage properties and relaxor behavior of lead-free $\mathrm{Ba} 1-\mathrm{x}$ Sm $2 x / 3 \mathrm{Zr} 0.15$ Ti 0.8503 ceramics. Dalton Trans 46: 14341-14347.

25.Saha S, Sinha TP (2002) Low-temperature scaling behavior of BaFe0.5Nb0.503. Phys Rev B 65: 134103.

26.Sahoo S, Dash U, Parashar SKS, Ali SM (2013) Frequency and temperature dependent electrical characteristics of $\mathrm{CaTiO} 3$ nano-ceramic prepared by high-energy ball milling. Journal of Advanced Ceramics 2: 291-300.

27.Sivakumar N, Narayanasamya A, Greneche JM, Murugaraj R, Lee YS (2010) Electrical and magnetic behaviour of nanostructured $\mathrm{MgFe} 2 \mathrm{O} 4$ spinel ferrite. Journal of Alloys and Compounds 504: 395-402. 